\title{
Diffraction measurement of plastic strain in A533B ferritic steel - a feasibility study
}

\author{
S. J. Lewis and C. E. Truman ${ }^{\mathrm{a}}$ \\ Department of Mechanical Engineering, University of Bristol, UK
}

\section{Introduction}

It is known that the physical properties of many engineering materials may be strongly affected by previous loading, in particular prior plastic deformation. Most obviously, work hardening will alter subsequent yielding behaviour. Plastic deformation may also preferentially align the material grain structure, resulting in anisotropy of subsequent behaviour and a change in material fracture resistance.

When physical characterisation is undertaken by experimental testing it is, therefore, important to have some knowledge of the current state of the material. As a result, it is desirable to have methods of quantitatively evaluating the level of plastic deformation which specimen material may have experienced prior to testing.

This paper presents the results of a feasibility study, using a ferritic reactor pressure vessel steel, into the use of diffractive methods for plastic strain evaluation. Using neutron diffraction, changes in diffraction peak width and anisotropy of peak response were correlated with plastic deformation in a tensile test. The relationships produced were then used to evaluate permanent deformation levels in large samples, representative of standard fracture toughness test specimens.

\section{Experimental measurements}

In order to investigate the effectiveness of two methods of plastic strain measurement (anisotropy strain and diffraction peak broadening [1]) it was necessary to manufacture suitable test specimens containing well defined plastic strain fields. Tensile test specimens were chosen as calibration specimens for the two techniques. In addition, a number of pre-strained 'blanks' for compact tension $(\mathrm{C}(\mathrm{T}))$ type fracture specimens were fabricated in order to compare with the data obtained from the tensile test samples. All samples were manufactured from A533B ferritic steel. This material was chosen as it is part of an ongoing study into the effects of plastic strain on fracture and it is typical of the type of steel commonly used in the power generation industry.

A number of tensile tests were undertaken, according to the ASTM E8 standard, and consistent stress-strain behaviour was recorded in all tests. The tensile test specimens were cylindrical, with diameter $8 \mathrm{~mm}$ and gauge length $80 \mathrm{~mm}$. To pre-strain the $\mathrm{C}(\mathrm{T})$ blanks, such that a uniform strain field was created with minimal residual stress, a number of large tensile-type specimens were manufactured. These were preloaded to the desired level of uni-axial plastic strain $(1 \%, 3 \%$ and $5 \%)$, determined according to clip gauges fixed to the centre of the specimens, before being cut into sections.

The specimens used were designed to provide the simplest possible, uni-axial plastic strain field in order to minimise the number of complicating factors when comparing the effectiveness of the two techniques. Neutron diffraction measurements were made using the ENGIN-X instrument on the ISIS neutron source at Rutherford Appleton laboratories, U.K.

\footnotetext{
a e-mail: c.e.truman@bristol.ac.uk
} 


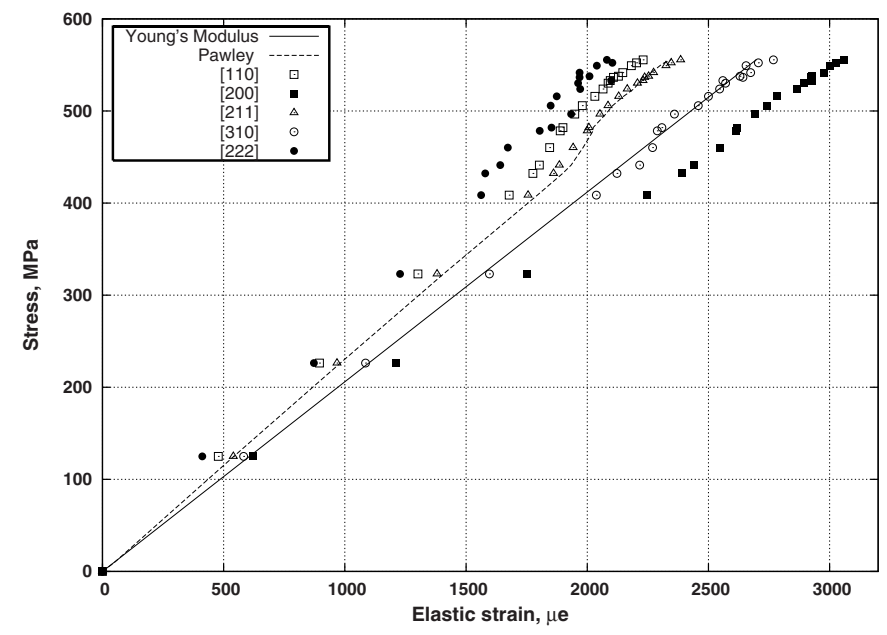

Fig. 1. Strain measurements from in-situ tensile test based on full Pawley refinement of the full diffraction spectra and analysis of individual peaks

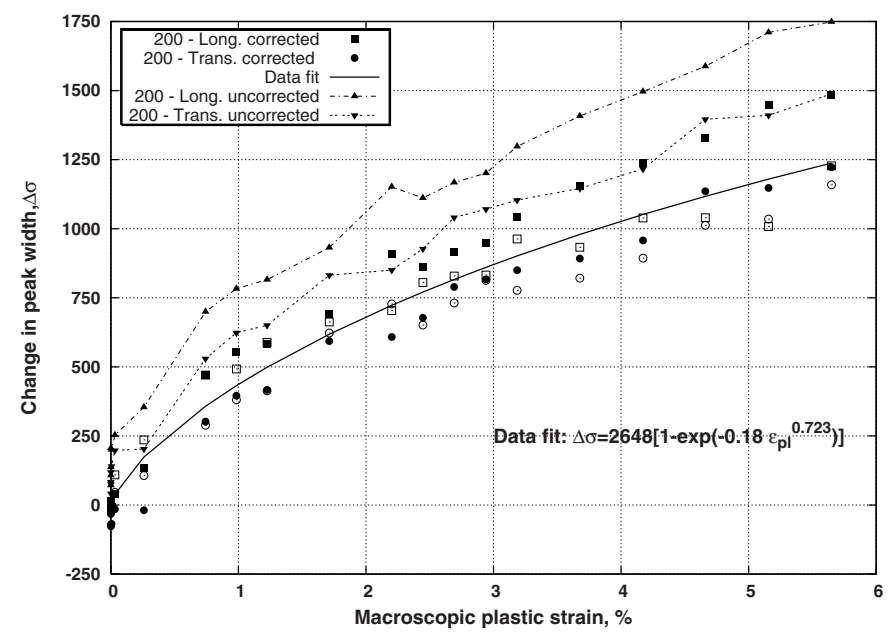

Fig. 2. Variation in [200] peak $\sigma$ component as a function of macroscopic plastic strain, as recorded during the in-situ tensile test. Open and closed symbols represent the loaded and unloaded states for each plastic strain level

\section{Results and comments}

Selected results are shown in Figures 1 and 2. From the results obtained it is apparent that both plastic anisotropy strain and diffraction peak broadening are strongly related to levels of macroscopic deformation. It would also appear that there are other influencing factors affecting their variation with the value of macroscopic plastic strain which inhibit accurate calibration and transference of relationships between these parameters from one specimen to another.

\section{References}

1. S. J. Lewis and C. E. Truman, Journal of Physics D, (under review) 